\title{
Probabilistic analysis of the minimum weighted flowtime scheduling problem
}

\author{
Alberto Marchetti Spaccamela \\ Universita di L'Aquila, Italy \\ Wan Soo Rhee \\ Ohio State University, Columbus, OH, USA \\ Leen Stougie \\ University of Amsterdam, Netherlands \\ Sara van de Geer \\ Centre for Mathematics and Computer Science, Amsterdam, Netherlands
}

Received July 1989

Revised November 1989

\begin{abstract}
The minimum weighted flow time scheduling problem is studied from a probabilistic point of view. A probability distribution is specified over its problem instances, and the asymptotics of the optimal solution value are derived. Rewriting this value as a $U$-statistic perturbed by a small term allows us to use results from the well-established theory on these statistics. We derive a law of large numbers, a law of the iterated logarithm and a central limit theorem. As a byproduct we obtain a proof of asymptotic optimality almost surely of a greedy heuristic (the shortest weighted processing time first rule) for the solution of the $N P$-complete problem with more than one machine.
\end{abstract}

minimum weighted flowtime schedule, strong law of large numbers, law of the iterated logarithm, central limit theorem, $U$-statistic, asymptotic optimality.

\section{Introduction}

In this paper we derive convergence properties of the optimal solution value of the scheduling problem with a minimum weighted flowtime objective. For this problem we are given $n$ jobs with processing times $p_{j}$ and weights $w_{j}, j=1, \ldots, n$, that are to be scheduled on $m$ identical parallel machines. Let $C_{j}$ be the completion time of job $j$, $j=1, \ldots, n$, under a feasible schedule. Then the weighted flow time of this schedule is $\sum_{j=1}^{n} w_{j} C_{j}$.

Correspondence to: prof. L. Stougie, Institute for Actuarial Sciences and Economics, University of Amsterdam, Jodenbreestraat 23, $1011 \mathrm{NH}$ Amsterdam, Netherlands.
This problem, in scheduling standard notation $P \| \sum w_{j} C_{j}$, is NP-hard for $m \geqslant 2$ (see Garey and Johnson, 1979, p. 240). For the single machine version $(m=1)$ an optimal schedule is obtained by the (polynomial) shortest weighted processing time first rule (SWPT) (Smith, 1956): the jobs are scheduled on the machine in order of increasing $p_{j} / w_{j}$-ratio.

In Section 2 we assume a probability distribution over the class of problem instances and analyse the asymptotic behaviour of the optimal solution value of the single machine problem. More specifically, we derive a strong law of large numbers which shows that the optimal solution value of problem instances with a growing number of 
jobs converges with probability $1(w p 1)$ to a constant, if properly normalized. Moreover we present a rate of convergence in the form of a law of the iterated logarithm, and a central limit theorem. The derivation of these results rely heavily on the transcription of the optimal solution value as a $U$-statistic perturbed by a term that is asymptotically negligible. The results follow then easily from the well-established theory on these statistics.

In Section 3 we consider the $m$-machine problem. We derive upper and lower bounds on the optimal solution value. For the upper bound we use the SWPT-rule as an approximation algorithm. Once having established these bounds, the asymptotics of the optimal solution value of the $m$-machine problem appear to come straightforward from those of the single machine problem. As a byproduct we obtain a proof of asymptotic optimality ( $w p 1)$ of the SWPT-rule.

Some concluding remarks follow in Section 4. Throughout the paper random variables are indicated by boldface characters.

\section{The single machine problem}

Let us assume that the processing times of the jobs $\boldsymbol{p}_{1}, \boldsymbol{p}_{2}, \ldots$ are positive, independent and identically distributed (i.i.d.) random variables and that the weights $\boldsymbol{w}_{1}, \boldsymbol{w}_{2}, \ldots$ are positive i.i.d. random variables. The optimal solution value of a minimum weighted flowtime problem is then a random variable denoted by $\boldsymbol{z}_{m, n}^{*}$.

In this section we study convergence properties of $z_{1, n}^{*}$, the optimal value of the single machine problem. This value can be characterized through the SWPT-rule (see Section 1). Let $\left(\boldsymbol{w}_{(1)}, \boldsymbol{p}_{(1)}\right),\left(\boldsymbol{w}_{(2)}, \boldsymbol{p}_{(2)}\right), \ldots,\left(\boldsymbol{w}_{(n)}, \boldsymbol{p}_{(n)}\right)$ be order statistics of $n$ observations of the weightprocessing time pair, where the order is dictated by increasing $\boldsymbol{p}_{j} / \boldsymbol{w}_{j}$-ratio's, i.e.,

$\frac{\boldsymbol{p}_{(1)}}{\boldsymbol{w}_{(1)}} \leqslant \frac{\boldsymbol{p}_{(2)}}{\boldsymbol{w}_{(2)}} \leqslant \cdots \leqslant \frac{\boldsymbol{p}_{(n)}}{\boldsymbol{w}_{(n)}}$.

The completion time of the $j$-th job in this sequence is equal to $\sum_{k=1}^{j} \boldsymbol{p}_{(k)}$, and the optimal value is given by

$\boldsymbol{z}_{1, n}^{*}=\sum_{j=1}^{n} \boldsymbol{w}_{(j)} \sum_{k=1}^{j} \boldsymbol{p}_{(k)}$.
To avoid analytical difficulties we assume that the ratio $p / w$ has a continuous distribution. In Section 2.1 we show that the behaviour of $z_{1, n}^{*}$, properly normalized, is essentially that of a socalled $U$-statistic. Given this knowledge, in Section 2.2 asymptotic properties of $z_{1, n}^{*}$ are derived almost straightforwardly from the theory on $U$ statistics.

\subsection{Towards a U-statistic}

Let $h\left(x_{1}, \ldots, x_{l}\right)$ be a symmetric real-valued function, called a kernel. Its domain is the $l$-fold Cartesian product of the spaces where the $x_{i}$ 's live on. For any such a kernel, the corresponding $U$-statistic, based on a sample of $n$ independent observations $\boldsymbol{X}_{1}, \ldots, \boldsymbol{X}_{n}$ on some distribution, with $n \geqslant l$, is obtained by averaging the kernel $h$ over the observations:

$\boldsymbol{U}_{n}=\frac{1}{\left(\begin{array}{c}n \\ l\end{array}\right)} \sum_{i_{1}, \ldots, i_{l}} h\left(\boldsymbol{X}_{i_{1}}, \ldots, \boldsymbol{X}_{i_{l}}\right)$

where the summation is over all the $\left(\begin{array}{l}n \\ 1\end{array}\right)$ possible combinations of $l$ out of $n$ observations. For a survey of the theory on $U$-statistics we refer to [Serfling 1980, Chapter 5].

In our specific situation we define the kernel function $h: \mathbb{R}^{2} \times \mathbb{R}^{2} \rightarrow \mathbb{R}$ as

$$
\begin{aligned}
& h\left((w, p),\left(w^{\prime}, p^{\prime}\right)\right) \\
& \quad= \begin{cases}\frac{1}{2} w p^{\prime} & \text { if } p / w>p^{\prime} / w^{\prime}, \\
\frac{1}{2} w^{\prime} p & \text { if } p / w<p^{\prime} / w^{\prime}, \\
0 & \text { if } p / w=p^{\prime} / w^{\prime} .\end{cases}
\end{aligned}
$$

The corresponding $U$-statistic is given by

$$
\boldsymbol{U}_{n}=\frac{2}{n(n-1)} \sum_{1 \leqslant i<j \leqslant n} h\left(\left(\boldsymbol{w}_{i}, \boldsymbol{p}_{i}\right),\left(\boldsymbol{w}_{j}, \boldsymbol{p}_{j}\right)\right) \text {. }
$$

It is not hard to see that $z_{1, n}^{*}$ in $(2.1)$ can be rewritten as

$$
\frac{z_{1, n}^{*}}{n(n-1)}=U_{n}+\frac{\sum_{j=1}^{n} \boldsymbol{w}_{j} \boldsymbol{p}_{j}}{n(n-1)} .
$$

We observe that $z_{1, n}^{*}$ normalized by $n(n-1)$ is a $U$-statistic perturbed by a normalized sum of i.i.d. random variables. The influence of the latter term on the asymptotic behaviour of $z_{1, n}^{*}$ will turn out to be negligible. 


\subsection{Convergence properties of $z_{l, n}^{*}$}

As a prerequisite for convergence theorems on $U$-statistics that we shall use, the variance of the conditional expectation $E\left(h\left((w, p),\left(w^{\prime}, p^{\prime}\right)\right) \mid\right.$ $(w, p))$ should not be equal to zero. Let us denote this conditional expectation by $h_{1}(w, p)$. Under this condition the theory of $U$-statistics resembles strongly that of sums of i.i.d. random variables. The condition is easily verified in our case if we assume that $(w, p)$ has a distribution whose support contains a two dimensional convex set.

Lemma 2.1. Under the above assumption and $E w_{1}$ $<\infty, E p_{1}<\infty$, we haie $\operatorname{Var}\left(h_{1}(w, p)\right)>0$.

Proof. If the lemma were not true, then there would exist constant $c$ such that $h_{1}(w, p)=$ $c(w p 1)$. Now take any $(w, p)$ and $(\alpha w, \alpha p)$ from the convex set in the support of the distribution of $(w, p)$, with $\alpha>0, \quad \alpha \neq 1$. Thus, we have $h_{1}(w, p)=h_{1}(\alpha w, \alpha p)=c$. However, from definition $(2.2)$ of $h\left((w, p),\left(w^{\prime}, p^{\prime}\right)\right)$ it is easy to see that $h_{1}(\alpha w, \alpha p)=\alpha h_{1}(w, p)$ a contradiction if $0<c<x$. But $h_{1}(w, p)=0$ (wp 1) can only occur if the distribution of $p / w$ is concentrated on only one point, which we excluded by our assumptions. Obviously, the assumptions $l$ iw $<x$, Ep $<x$ imply $h_{1}(w, p)<x($ wp 1$)$.

Now we are ready $t()$ apply directly theorems from Serfling (1980), (hapter 5) to establish respectively, a law of large numbers, a law of the iterated logarithm, and a central limit theorem for $z_{1, n}^{*} /(n(n-1))$. I cet $\theta=\operatorname{lih}\left((w, p),\left(w^{\prime}, p^{\prime}\right)\right)$. E.g. if $p$ and $w$ are uniformly distributed over $[0,1]$, then tedious calculations lead to $\theta=7 / 108$.

Theorem 2.2 (Law of large numbers). If $E w_{1}<x$, $E p_{1}<x$ and $E w_{1} p_{1}<x$, then

$\lim _{n \rightarrow \infty} \frac{z_{1, n}^{*}}{n(n-1)}=\theta \quad($ wp 1$)$.

Proof. Recall formula (2.3). Using the independence of $(w, p)$ and $\left(w^{\prime}, p^{\prime}\right)$ we have

$$
\begin{aligned}
\theta & =E\left(h\left((w, p),\left(w^{\prime}, p^{\prime}\right)\right)\right)<\frac{1}{2} E\left(w p^{\prime}+w^{\prime} p\right) \\
& \leqslant \frac{1}{2} E w L^{\prime}+\frac{1}{2} E w^{\prime} E p<x
\end{aligned}
$$

by the assumptions. Hence Theorem A from Serfling (1980, p. 190) can be applied to prove that

$$
\lim _{n \rightarrow \infty} U_{n}=\theta \quad(\text { wp } 1) \text {. }
$$

Since $E w_{1} p_{1}<\infty$, the usual strong law of large numbers implies that

$\lim _{n \rightarrow \infty} \frac{\sum_{j-1}^{n} \boldsymbol{w}_{j} \boldsymbol{p}_{j}}{n(n-1)}=0 \quad$ (wp 1).

Together (2.4) and (2.5) imply the theorem.

Theorem 2.3 (Law of the iterated logarithm). Let $\sigma^{2}=\operatorname{Var}\left(h_{1}(w, p)\right)$. Assume that $\sigma^{2}>0, E w_{1}^{2}<x$ and $E p_{1}^{2}<\infty$. Then

$$
\limsup _{n \rightarrow \infty} \frac{\sqrt{n}\left(z_{1, n}^{*} /(n(n-1))-\theta\right)}{2 \sigma(2 \log \log n)^{1 / 2}}=1 \quad \text { (wp 1). }
$$

Proof. Recall formula (2.3). By independence of $(w, p)$ and $\left(w^{\prime}, p^{\prime}\right)$ we have

$$
\begin{aligned}
E\left(\left(h^{2}(w, p),\left(w^{\prime} p^{\prime}\right)\right)\right) & \leqslant{ }_{4}^{1} E\left(w^{2} p^{\prime 2}+w^{\prime 2} p^{2}\right) \\
& <x .
\end{aligned}
$$

Therefore, we may apply 'Theorem C' from Serfling (1980, p. 191) to obtain

$$
\limsup _{n \rightarrow \infty} \frac{\sqrt{n\left(U_{n}-\theta\right)}}{2 \operatorname{co}(2 \log \log n)^{1 / 2}}=1 \quad(\text { wp 1). }
$$

By Cauchy-Schwartz' inequality we have

$E w_{1} p_{1}<\left(E w_{1}^{2} E p_{1}\right)^{1 / 2}<x$.

so that the strong law of large numbers implies that for every $\delta>0$

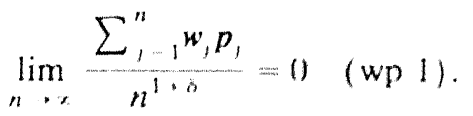

Together (2.7) and (2.8) imply the theorem.

Theorem 2.4 (central limit theorem). Let or $\operatorname{Var}\left(h_{1}(w, p)\right)$. Assume that $\sigma^{2}-\left(1, E w_{1}^{2}-x_{2}, F_{1}^{2}\right.$ $x$. The'n

$$
\frac{\sqrt{ } n}{2 \sigma}\left(\frac{z_{1, n}^{*}}{n(n-1)}-\theta\right) \rightarrow \mathrm{N}(0,1) \text {. }
$$

Proof. Since we have the same assumptions here as in Theorem 2.3, (2.6) holds. Therefore we may 
apply Theorem A from Serfling (1980 p. 192) to obtain

$\frac{\sqrt{n}}{2 \sigma}\left(U_{n}-\theta\right) \rightarrow \mathrm{N}(0,1)$.

This together with (2.8), which holds here too, implies the theorem.

\section{The $m$-machine problem}

The stochastic assumptions and the notation of the previous section are maintained here. In addition, we assume that the number of machines is fixed.

\subsection{Upper and lower bounds on $z_{m, n}^{*}$}

For a lower bound on $z_{m, n}^{*}$ we refer to Eastman et al. (1964): for any schedule of the jobs, such that on each machine the jobs assigned to it are scheduled in order of increasing $p / w$-ratio, the sum of the weighted completion times is bounded from below by

$\frac{m-1}{2 m} \sum_{j=1}^{n} \boldsymbol{w}_{j} \boldsymbol{p}_{j}+\frac{1}{m} \boldsymbol{z}_{1, n}^{*}$.

Simple exchange arguments show that any optimal schedule must satisfy this property. Hence,

$z_{m, n}^{*} \geqslant \frac{m-1}{2 m} \sum_{j=1}^{n} \boldsymbol{w}_{j} \boldsymbol{p}_{j}+\frac{1}{m} z_{1, n}^{*}$

For an upper bound we consider the before mentioned SWPT-rule as an approximation method for the solution of the $m$-machine problem. Every next job to be scheduled is assigned to the machine that is first available. Switching for once again to order statistics (see Section 2), we notice that the $j$-th job to be scheduled will start no later than $(1 / m) \sum_{k=1}^{j-1} p_{(k)}, j=1, \ldots, n$. Hence, its weighted completion time is bounded from above by

$(1 / m) \boldsymbol{w}_{(j)} \sum_{k=1}^{j-1} \boldsymbol{p}_{(k)}+\boldsymbol{w}_{(j)} \boldsymbol{p}_{(j)}, \quad j=1, \ldots, n$.

Summation over $j$ yields the following upper bound on the value of the solution produced by the SWPT-rule, denoted by $z_{m, n}^{\mathrm{SWPT}}$, which is on its turn an upper bound on $z_{m, n}^{*}$.

$$
\begin{aligned}
\boldsymbol{z}_{m, n}^{*} & \leqslant z_{m, n}^{\mathrm{SWPT}} \leqslant \frac{1}{m} \sum_{j=1}^{n} \boldsymbol{w}_{(j)} \sum_{k=1}^{j-1} \boldsymbol{p}_{(k)}+\sum_{j=1}^{n} \boldsymbol{w}_{(j)} \boldsymbol{p}_{(j)} \\
& =\frac{1}{m} \sum_{j=1}^{n} \boldsymbol{w}_{(j)} \sum_{k=1}^{j} \boldsymbol{p}_{(k)}+\frac{m-1}{m} \sum_{j=1}^{n} \boldsymbol{w}_{(j)} \boldsymbol{p}_{(j)} .
\end{aligned}
$$

Since the first term on the right hand side is equal to $(1 / m) z_{1, n}^{*}$ (see (2.1)), we may rewrite the above inequality as

$\boldsymbol{z}_{m, n}^{*} \leqslant \boldsymbol{z}_{m, n}^{\mathrm{SWPT}} \leqslant \frac{1}{m} \boldsymbol{z}_{1, n}^{*}+\frac{m-1}{m} \sum_{j=1}^{n} \boldsymbol{w}_{j} \boldsymbol{p}_{j}$.

We see that both the upper and the lower bound consists of a term $(1 / m) z_{1, n}^{*}$ and a term including $\sum_{j=1}^{n} \boldsymbol{w}_{j} \boldsymbol{p}_{j}$, which in Section 2.2 turned out to become negligible asymptotically.

\subsection{Convergence properties of $z_{m, n}^{*}$}

From the upper and lower bound (3.2) and (3.1) it is clear that the asymptotics of $z_{m, n}^{*}$ follow those of $(1 / m) z_{1, n}^{*}$. Therefore we give the following convergence theorems for $z_{m, n}^{*}$ without proof.

Theorem 3.1 (Law of large numbers). If $E \boldsymbol{w}_{1}<\infty$, $E \boldsymbol{p}_{1}<\infty$ and $E \boldsymbol{w}_{1} \boldsymbol{p}_{1}$, then

$\lim _{n \rightarrow \infty} \frac{z_{m, n}^{*}}{n(n-1)}=\frac{\theta}{m} \quad$ (wp 1$)$.

Theorem 3.2 (Law of the iterated logarithm). Let $\sigma^{2}=\operatorname{Var}\left(h_{1}(\boldsymbol{w}, \boldsymbol{p})\right)$. If $E \boldsymbol{w}_{1}^{2}<\infty, E \boldsymbol{p}_{1}^{2}<\infty$ and $\sigma^{2}$ $>0$, then

$\limsup _{n \rightarrow \infty} \frac{m \sqrt{n}\left(\frac{z_{m, n}^{*}}{n(n-1)}-\theta / m\right)}{2 \sigma(2 \log \log n)^{1 / 2}}=1 \quad($ wp 1).

Theorem 3.3 (Central limit theorem). Let $\sigma^{2}=$ $\operatorname{Var}\left(h_{1}(\boldsymbol{w}, \boldsymbol{p})\right)$. If $E \boldsymbol{w}_{1}^{2}<\infty, E \boldsymbol{p}_{1}^{2}<\infty$ and $\sigma^{2}>0$, then

$\frac{m \sqrt{n}}{2 \sigma}\left(\frac{z_{m, n}^{*}}{n(n-1)}-\frac{\theta}{m}\right) \leadsto \mathrm{N}(0,1)$.

All of the above holds if we substitute $z_{m, n}^{\text {SWPT }}$ for $z_{m, n}^{*}$. This implies among other things asymptotic 
optimality (wp 1) of the SWPT-rule for the minimum weighted flowtime scheduling problem:

$$
\lim _{n \rightarrow \infty} \frac{z_{m, n}^{\mathrm{SWPT}}-z_{m, n}^{*}}{z_{m, n}^{*}}=0 \quad(\text { wp } 1)
$$

\section{Concluding remarks}

The results presented in this paper generalize and extend results on the unweighted minimum flowtime problem in Frenk et al. (1984). We notice that it is also possible to obtain the results using the entropy approach to empirical process theory (cf. Pollard, 1984). The results in Section 3 indicate nice behaviour of the SWPT-rule as an approximation method for the $m$-machine problem. It complements results on the worst-case behaviour of this method presented in Weiss et al. (1987).

\section{References}

W.L. Eastman, S. Even and I.M. Isaacs (1964), "Bounds for the optimal scheduling of $n$ jobs on $m$ processors", Management Sci. 11, 268-279.

J.B.G. Frenk, A.H.G. Rinnooy Kan and L. Stougie (1984), A hierarchical scheduling problem with a well-solvable second stage. Annals of Oper. Res. 1, 43-58.

M.R. Garey and D.S. Johnson (1979), Computers and Intractability: A guide to the theory of NP-Completeness, Freeman, San Francisco, CA.

D. Pollard (1984), Convergence of Stochastic Processes, Springer Series in Statistics, Springer-Verlag, New York.

R.J. Serfling (1980), Approximation Theorems of Mathematical Statistics, Wiley, New York.

W.E. Smith (1956), "Various optimizers for single-stage production", Naval Res. Logist. Quart. 3, 59-66.

G. Weiss, C.C. Huang, C.L. Li, S. Liu, M. Pinedo and J. Song (1987), "A worst-case analysis of Smith's rule for scheduling parallel machines to minimize weighted flow time", Georgia Tech. ISYE technical report. 